\title{
Existence and Stability of Foliations by $J$-Holomorphic Spheres
}

\author{
R. Hind J. von Bergmann
}

September 19, 2021

\begin{abstract}
We study the existence and stability of holomorphic foliations in dimension greater than 4 under perturbations of the underlying almost complex structure. An example is given to show that, unlike in dimension $4, J$-holomorphic foliations are not stable under large perturbations of almost complex structure.
\end{abstract}

\section{Introduction}

The theory of pseudoholomorphic curves was introduced in Gromov's seminal paper [Gro85]. There is a Fredholm theory showing that, for generic almost-complex structures $J$, pseudoholomorphic, or $J$-holomorphic, curves appear in finite dimensional families, with the dimension given by the Riemann-Roch theorem. Furthermore, in the presence of a taming symplectic form, suitable moduli spaces of $J$-holomorphic curves are compact modulo bubbling. These results have many important applications in symplectic topology. Notably they lead to Gromov-Witten invariants and Floer homology, which have been the main methods for establishing rigidity results in symplectic and contact topology.

Applied to symplectic manifolds of dimension 4 the theory of pseudoholomorphic curves is especially powerful and it becomes possible to prove classification results which are as yet inaccessible in higher dimensions. For example, symplectic forms on $S^{2} \times S^{2}$ are classified, see [Tau96] and [Gro85, their symplectomorphism groups are well understood, see Gro85] and [AM00], and the Lagrangian spheres are all known to be symplectically equivalent, see [Hin04. These results all rely on the existence of foliations by $J$-holomorphic spheres. More precisely, they utilize the following theorem of Gromov. We say that a homology class $A \in H_{2}(X)$ is $\omega$-minimal if $\omega(A)=\min _{B \in H_{2}(X), \omega(B)>0} \omega(B)$.

Theorem 1.1. Let $(X, \omega)$ be a symplectic 4-manifold with a tamed almost-complex structure $J$ and suppose that there exists an embedded symplectic sphere in a homology class $A$ satisfying $A \bullet A=0$ and $A$ is $\omega$-minimal.

Then $X$ is foliated by the images of J-holomorphic spheres homologous to A. The foliations vary smoothly with the almost-complex structure $J$.

The aim of paper is to investigate the extent to which this remains true when $X$ has higher dimension. 
It turns out that in general Theorem 1.1 is false if $X$ is allowed to have dimension greater than 4. The existence of $J$-holomorphic spheres in the class $A$ can be guaranteed at least for an open set of almost-complex structures by imposing an index constraint. But even if a foliation is known to exist for a particular $J$, it is unstable in the sense that varying $J$, even in the most generic fashion, can cause the foliation to degenerate.

To be more precise, we recall that given a family of tame almost-complex structures $J_{t}$, $0 \leq t \leq 1$, on the symplectic manifold $X^{2 n}$ we can define the universal moduli space

$$
\mathcal{M}=\left\{(u, t) \mid u: S^{2} \rightarrow X J_{t} \text {-holomorphic, }\left[u\left(S^{2}\right)\right]=A\right\} .
$$

Suppose that $c_{1}(A)=2$, then $\mathcal{M}$ has virtual dimension $2 n+5$ and if the family $\left\{J_{t}\right\}$ is regular then $\mathcal{M}$ is a manifold (with boundary) of dimension $2 n+5$. Furthermore, in the generic case the projection map $T: \mathcal{M} \rightarrow[0,1],(u, t) \mapsto t$ is a Morse function and for all but finitely many $t$ the fiber $\mathcal{M}_{t}$ is a manifold of dimension $2 n+4$ consisting of the $J_{t}$-holomorphic spheres in the class $A$. For such regular $t$ there is a smooth evaluation map

$$
e_{t}: \mathcal{M}_{t} \times_{G} S^{2} \rightarrow X
$$

where the equivalence relation $G$ is reparameterization of the holomorphic spheres. Both $\mathcal{M}_{t} \times_{G} S^{2}$ and $X$ are smooth $2 n$-dimensional manifolds.

Definition 1.2. We say that $X$ is foliated by $J_{t}$-holomorphic spheres in the class $A$ if the map $e_{t}$, when restricted to some connected component of its domain, is a homeomorphism.

We say that $X$ is smoothly foliated by $J_{t}$-holomorphic spheres in the class $A$ if the map $e_{t}$, when restricted to some connected component of its domain, is a diffeomorphism.

When $X$ is 4-dimensional, or when the almost-complex structure $J_{t}$ is integrable, these two notions coincide, but in higher dimensions there exist foliations (at least if we allow nonregular curves) for which the corresponding evaluation map is a smooth homeomorphism that is not a diffeomorphism. An example is given in Remark 2.5.

The following result shows that Theorem 1.1 fails completely in dimension greater than four. Let $\left(M, \omega_{M}\right)$ be a symplectic manifold of dimension at least four.

Theorem 1.3. There exists a regular family $J_{t}$ of tame almost-complex structures on $(X, \omega)=$ $\left(S^{2} \times M, \sigma_{0} \oplus \omega_{M}\right)$ such that $\mathcal{M}$ has a component $\mathcal{N}$ where the curves in $t^{-1}(0) \cap \mathcal{N}$ form a foliation of $X$ but the curves in $t^{-1}(1) \cap \mathcal{N}$ do not, the curves are not disjoint.

In fact, we can take $J_{0}$ to be a product structure on $S^{2} \times M$ and so $\mathcal{M}_{0}$ has a single component consisting of curves with images $S^{2} \times\{z\}$ for $z \in M$. Fixing a point $0 \in M$ we can further assume that the corresponding sphere $C_{0}=S^{2} \times\{0\}$ is $J_{t}$-holomorphic and regular for all $t$. However there exists a two parameter family of curves $C_{r}$ in $\mathcal{M}_{1}$ which includes $C_{0}$ but with $C_{r} \cap C_{0} \neq \emptyset$ for all $r$.

An analog of Theorem 1.1 does remain true if we impose restrictions on the $J_{t}$. In this paper we will explain how to guarantee the existence and stability of foliations in the case of integrable complex structures and additional restrictions on the curvature.

Theorem 1.4. Let $(X, \omega, J)$ be Kähler with holomorphic bisectional curvature bounded from below by $c>-\pi / \omega(A)$, where $A \in H_{2}(X ; \mathbb{Z})$ is an $\omega$-minimal homology class with $G W_{0,1, A}^{X}(p t)=$ 1. Then $X$ is smoothly foliated by $J$-holomorphic spheres. 
We remark that if $X$ is a product $(M, k \omega) \times\left(S^{2}, \sigma\right)$, where $(M, \omega, J)$ is Kähler and $\sigma$ is the area form on $S^{2}$, then a product complex structure will satisfy the hypotheses of Theorem 1.4 whenever $k$ is sufficiently large, and so will any other integrable complex structure that is sufficiently close to the product one.

Of central importance to the stability of foliations is the notion of superregularity as defined in Don02].

Definition 1.5. A real-linear Cauchy-Riemann operator $D$ on a complex vector bundle over $S^{2}$ is called regular if $D$ is surjective. It is called superregular if $\operatorname{ker} D$ contains a collection of sections that are linearly independent over each point in $S^{2}$. A choice of such a collection of sections is called a superregular basis for $D$.

A $J$-holomorphic sphere $u$ is called regular if the induced real-linear Cauchy-Riemann operator $D_{u}$ on $u^{*} T X$ is regular. An immersed $J$-holomorphic sphere $u$ is called superregular if $D_{u}$ acting on sections of the normal bundle is superregular.

Note that regularity does not imply superregularity and vice versa. For example, no regular linearized operator at a $J$-holomorphic curve in a 4 -manifold with self-intersection number $\neq 0$ is superregular. We will give an example of a superregular operator that is not regular in Section 2.1.

Another way to understand what it means for a linearized operator to be regular and superregular is the following. Suppose $u$ is regular so that the moduli space of curves near $u$ is a smooth manifold. Then, in the language of Section 3.4 in [MS04, the evaluation map from the moduli space of $J$-holomorphic curves near a map $u: S^{2} \rightarrow X$ is transverse to all $x \in$ image $(u) \subset X$ if and only if $u$ superregular. Hence all curves $u$ in a smooth foliation are superregular.

The paper is arranged as follows. We first establish the non-existence result Theorem 1.3 in Section 2, Then we discuss the integrable case in Section 3 to prove Theorem 1.4.

\section{Non-stability of foliations}

For clarity of exposition we will restrict ourselves to work in dimension 6 . It is clear how to generalize this to higher dimension, e.g. by taking the product with another symplectic manifold with compatible almost complex structure. However, our construction does not work in dimension less than 6 since in that case Hirsch's theorem about immersions does not apply, and consequently Lemma 2.4 does not hold.

\subsection{Superregular Operator with Cokernel}

Here we will construct a superregular Cauchy-Riemann operator with nontrivial cokernel. This immediately gives examples of foliations by holomorphic spheres which are not superregular.

Throughout this section $N=S^{2} \times \mathbb{R}^{4}$ denotes the trivial bundle. Let $\left\{\bar{e}_{i}\right\}_{i=1}^{4}$ be the canonical basis of $\mathbb{R}^{4}$ and $J_{0}$ the canonical complex structure. Using the trivialization of $N$ we will frequently identify sections of $N$ with functions from $S^{2}$ into $\mathbb{R}^{4}$.

Recall the structure of a real-linear Cauchy-Riemann $D$ operator acting on sections $\xi$ of the complex vector bundle $\left(N, J_{0}\right)$ with trivial connection $\nabla$ via

$$
D \xi=\frac{1}{2}\left(\nabla \xi+J_{0} \nabla \xi \circ j\right)+\frac{1}{2} Y \xi=\bar{\partial}_{J_{0}} \xi+\frac{1}{2} Y \xi
$$


where $Y: N \rightarrow \Lambda^{0,1}\left(T^{*} S^{2} \otimes_{\mathbb{C}} N\right)$ is a vector bundle homomorphism.

Recall Definition 1.5 of our use of the terms regular and superregular.

Lemma 2.1. Let $D$ be a superregular real-linear Cauchy-Riemann operator on $\left(N, J_{0}\right)$ with superregular basis $\left\{e_{i}\right\}_{i=1}^{4}$. Let $\Phi: S^{2} \times \mathbb{R}^{4} \rightarrow N$ be the corresponding trivialization, i.e. $\Phi(z, x)=\sum_{i=1}^{n} x_{i} e_{i}(z)$.

Then for a function $f: S^{2} \rightarrow \mathbb{R}^{4}$ we have $D \Phi(z, f(z))=\Phi_{*} \bar{\partial}_{J} f$, where $J: S^{2} \rightarrow \operatorname{End}\left(\mathbb{R}^{4}\right)$, is given by $\Phi^{*} J_{0}$.

Proof.

$$
D \Phi(z, f(z))=D \sum_{i=1}^{4} f_{i}(z) e_{i}(z)=\sum_{i=1}^{4} \frac{1}{2}\left\{d f_{i}+J_{0} d f_{i} \circ j\right\} e_{i}=\Phi_{*}\left(\bar{\partial}_{J} f\right) .
$$

We need the following elementary observation.

Lemma 2.2. Given any four sections $\left\{e_{i}\right\}_{i=1}^{4}$ of $N=S^{2} \times \mathbb{C}^{2}$ that are linearly independent over each $z \in S^{2}$, there exists a unique real-linear Cauchy-Riemann operator $D=\bar{\partial}_{0}+Y$, where $\bar{\partial}_{0}$ is the canonical complex Cauchy-Riemann operator, $Y \in \Lambda^{0,1} T^{*} S^{2} \otimes \mathbb{C}^{2}$, and $\left\{e_{i}\right\}_{i=1}^{4}$ is a superregular basis for $D$, i.e. so that $D e_{i}=0$ for $i=1, \ldots, 4$.

Proof. Let $\nu_{i}=D_{0} e_{i}$. Since the $\left\{e_{i}\right\}_{i=1}^{4}$ are linearly independent for all $z \in S^{2}$ we may define $Y$ via $Y_{z} e_{i}(z)=-\nu_{i}(z)$. Thus $D e_{i}=0$ so $\left\{e_{i}\right\}_{i=1}^{4}$ is a superregular basis. Conversely, if $D e_{i}=0$ for $i=1, \ldots, 4$ then $Y_{z}\left(e_{i}(z)\right)=-\nu_{i}(z)$, defining $Y$ uniquely.

We now aim to construct a superregular real-linear Cauchy-Riemann operator on $N$ that has a non-trivial cokernel. The following Lemma clears some topological obstructions.

Lemma 2.3. There exists a complex bundle monomorphism $F_{0}: T S^{2} \rightarrow \underline{\mathbb{C}^{2}}$.

Here $\underline{\mathbb{C}^{2}}$ denotes the trivial $\mathbb{C}^{2}$-bundle over $S^{2}=\mathbb{C P}^{1}$.

Proof. Consider the diagram in Figure 2.1. Let $K \rightarrow \mathbb{C P}^{1}$ be the tautological bundle, i.e.

$$
K=\left\{\left(v,[z: w] \mid v \in \operatorname{span}_{\mathbb{C}}(z, w), \quad(z, w) \in \mathbb{C}^{2}\right\}\right.
$$

and let $\tilde{f}_{0}: S^{2} \rightarrow \mathbb{C P}^{1}$ be a degree 2 map. Then $T S^{2}$ and $\tilde{f}_{0}^{*} K$ have the same Chern class, so they are isomorphic complex vector bundles. Let $\tilde{F}_{0}: T S^{2} \rightarrow K$ be a bundle isomorphism (covering $\tilde{f}_{0}$ ).

Let $\iota: K \rightarrow \underline{\mathbb{C}^{2}}$ be the standard inclusion, i.e. $\iota(v,[z: w])=(v,[z: w]) \in \mathbb{C}^{2} \times \mathbb{C P}^{1}=\underline{\mathbb{C}^{2}}$ and set

$$
F_{0}: T S^{2} \rightarrow \underline{\mathbb{C}^{2}}, \quad F_{0}=\iota \circ \tilde{F}_{0}
$$

$F_{0}$ is an injective complex vector bundle homomorphism because $\tilde{F}_{0}$ and $\iota$ are. 


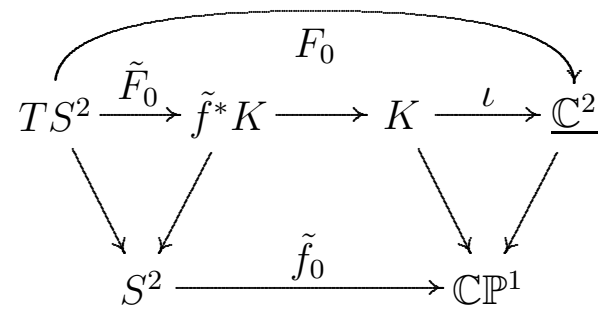

Figure 1: The construction of the map $F_{0}$.

Set $f_{0}: S^{2} \rightarrow \mathbb{C}^{2}, f_{0}(z)=0$ and let $F_{0}: T S^{2} \rightarrow f_{0}^{*} T \mathbb{C}^{2}=\underline{\mathbb{C}^{2}}$ as in Lemma 2.3. We aim to construct an actual immersion $f_{1}: S^{2} \rightarrow \mathbb{R}^{4}$ so that $\left(f_{1}, F_{1}=d f_{1}\right)$ has the same topological data as $\left(f_{0}, F_{0}\right)$.

By Theorem 6.1 of [Hir59] (or alternatively by the $h$-principle) there exists an immersion $f_{1}: S^{2} \rightarrow \mathbb{R}^{4}$ with $F_{1}=d f_{1}: S^{2} \rightarrow f_{1}^{*} T \mathbb{R}^{4}=\underline{\mathbb{R}^{4}}$ together with a homotopy $f_{t}: S^{2} \rightarrow$ $\mathbb{R}^{4}$ connecting $f_{0}$ and $f_{1}$ covered by a homotopy of (real) monomorphisms $F_{t}: T S^{2} \rightarrow \underline{\mathbb{R}^{4}}$ connecting $F_{0}$ and $F_{1}$. Here $\mathbb{R}^{4}$ is again the trivial bundle and we implicitly made use of the canonical (real) isomorphism $\underline{\mathbb{C}^{2}}=\underline{\mathbb{R}^{4}}$.

We need one more definition to construct a superregular operator with non-trivial cokernel. Let $\langle\cdot, \cdot\rangle$ denote the standard inner product on $\mathbb{R}^{4}$ and let $\mathcal{J}$ denote the space of complex structures on $\mathbb{R}^{4}$ and let $\mathcal{A}$ be the set of injective (real-linear) homomorphisms from $\mathbb{C}$ into $\mathbb{R}^{4}$. We define the map

$$
\Phi: \mathcal{A} \rightarrow \mathcal{J}
$$

in the following way. $A$ defines a splitting $\mathbb{R}^{4}=V \oplus W$, where $V=$ image $A$ and $W=V^{\perp}$. Define $J=\Phi(A)$ to be the unique $J \in \mathcal{J}$ that leaves this splitting invariant, makes $A$ complex linear, and satisfies

$$
\left\langle J e_{1}, e_{2}\right\rangle=1, \quad\left\langle J e_{1}, e_{1}\right\rangle=0
$$

on an oriented orthonormal basis $e_{1}, e_{2}$ of $W$.

Lemma 2.4. There exists a superregular real-linear Cauchy-Riemann operator D with nontrivial cokernel.

Proof. For each $z \in S^{2}$ define $J(z)=\Phi \circ F_{1}(z)$, where $\Phi$ is the map from Equation (2). Note that $\Phi \circ F_{0}(z)=J_{0}$, so $\Phi \circ F_{0}$ is covered by the map $G_{0}=\operatorname{Id}: S^{2} \rightarrow G L(4, \mathbb{R})$, where the projection $\pi: G L(4, \mathbb{R}) \rightarrow \mathcal{J}$ is given by $\pi g=g^{*} J_{0}=g^{-1} \circ J \circ g$. The map $\pi: G L(4, \mathbb{R}) \rightarrow \mathcal{J}$ is a bundle projection and thus has the homotopy lifting property. Let $G_{t}$ be a lift of the homotopy $\Phi \circ F_{t}$ to $G L(4, \mathbb{R})$.

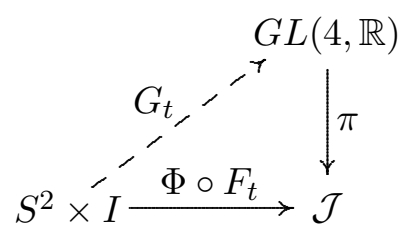

For $i=1, \ldots, 4$ define sections $e_{i}(z)=G_{1}(z) \bar{e}_{i}$, where $\left\{\bar{e}_{i}\right\}_{i=1}^{4}$ denotes the canonical basis of $\mathbb{R}^{4}$. By definition these are linearly independent for each $z \in S^{2}$, so by Lemma 2.2 we can 
choose $Y$ so that these sections satisfy $D e_{i}=0$, where $D=D_{0}+Y$. Thus $D$ is superregular with superregular basis $\left\{e_{i}\right\}_{i=1}^{4}$.

Set $e_{5}(z)=G_{1}(z) f_{1}(z)$, and note that $\bar{\partial}_{J(z)} f_{1}=0$ by the definition of $J(z)$. So by Lemma $2.1 e_{5}$ satisfies $D e_{5}=0$.

Thus the kernel of $D$ is at least 5 -dimensional, so $D$ has non-trivial cokernel.

Remark 2.5. Adding a suitable multiple of $e_{4}$ to $e_{5}$ we may assume that the pointwise inner product $\left\langle e_{4}(z), e_{5}(z)\right\rangle \geq 0$ but that the strict inequality does not hold. Then consider the map

$$
\begin{gathered}
e: \mathbb{R}^{4} \times S^{2} \rightarrow N \\
\left(t_{1}, t_{2}, t_{3}, t_{4}, z\right) \mapsto \sum_{i=1}^{3} t_{i} e_{i}+t_{4} e_{5}+t_{4}^{2} e_{4} .
\end{gathered}
$$

This is clearly a smooth map giving a foliation of $N$ but its differential is not an isomorphism wherever $z$ satisfies $\left\langle e_{4}(z), e_{5}(z)\right\rangle=0$.

\subsection{Family of Almost Complex Structures}

Here we will extend the example from the previous section to construct a family $D_{s}$ of CauchyRiemann operators for $s \in[-1,2]$ such that $D_{s}$ is regular for all $s$, superregular for $s$ close to 2 , but not superregular for $s$ close to -1 . The example can be globalized to produce the counterexample needed for Theorem [1.3.

Let $N \rightarrow S^{2}$ be a trivial complex rank 2 vector bundle. Fix an inner product $\langle\cdot, \cdot\rangle$ on $\mathrm{N}$ and let

$$
D: \Gamma N \rightarrow \Omega^{0,1}(N)
$$

be a superregular real-linear Cauchy-Riemann operator with non-trivial cokernel as given by Lemma 2.4. Let $K=\operatorname{ker}(D)$ and fix a superregular basis $\left\{e_{i}\right\}_{i=1}^{4}$ and let $e_{5} \in K$ be another section that is linearly independent from the $\left\{e_{i}\right\}_{i=1}^{4}$. Without loss of generality assume that $e_{5}$ is perpendicular to $e_{1}, e_{2}, e_{3}$ in $L^{2}$ and the pointwise inner product $h(z)=\left\langle e_{4}(z), e_{5}(z)\right\rangle \geq 0$ with $h(0)=0$. Assume that $\left\|e_{i}\right\|=1, i=1, \ldots, 4$ and scale $e_{5}$ so that there exists $p_{0} \in S^{2}$ with

$$
h\left(p_{0}\right)=\left\langle e_{4}\left(p_{0}\right), e_{5}\left(p_{0}\right)\right\rangle=1 .
$$

Let $C=\operatorname{coker}(D)$ be spanned by the orthonormal basis $\left\{\eta_{i}\right\}_{i=1}^{n} \in C$. Let $\tilde{D}: K^{\perp} \rightarrow C^{\perp}$ be the restriction of $D$ to $K^{\perp}$ and let $P: C^{\perp} \rightarrow K^{\perp}$ denote its inverse. Let

$$
\pi_{C}: \Omega^{0,1}(N) \rightarrow C
$$

denote the orthogonal projection in $L^{2}$.

Lemma 2.6. There exists family of vector bundle homomorphisms $Y_{s}: N \rightarrow \Lambda^{0,1}\left(T^{*} S^{2} \otimes_{\mathbb{C}} N\right)$, $s \in[-1,1]$ so that

$$
L_{s}=\pi_{C} \circ Y_{s}: \Gamma(N) \rightarrow C
$$

is surjective and

$$
K_{s}=\operatorname{ker}\left(L_{s}\right) \cap K=\operatorname{span}\left\{e_{1}, e_{2}, e_{3}, s e_{4}+(1-s) e_{5}\right\}
$$


In order to prove this we need the following three lemmas. We will make use of the notation introduced above. Also recall the point $p_{0} \in S^{2}$ from Equation (3), so the family of sections $e_{1}^{s}$ defined via $e_{i}^{s}=e_{i}$ for $i=1, \ldots, 3$ and $e_{4}^{s}=s e_{4}+(1-s) e_{5}$ are linearly independent vectors over $p_{0}$ for all $s \in[-1,1]$. Let $U \subset S^{2}$ be an open neighborhood of $p_{0}$ so that the $\left\{e_{i}^{s}(z)\right\}_{i=1}^{4}$ still are linearly independent vectors over all $z \in \bar{U}$. Let $V \subset C^{\perp}$ denote the subspace of smooth sections in $C^{\perp}$ that are supported in $U$. Then we can define a family of homomorphisms $Y_{s}$ from $N$ to $\Lambda^{0,1}\left(T^{*} S^{2} \otimes_{\mathbb{C}} N\right)$ via functions $g_{i}^{s}:[-1,1] \times U \rightarrow V$ by

$$
Y_{s}\left(e_{i}^{s}\right)=g_{i}^{s} .
$$

Set $L_{s}=\pi_{C} \circ Y_{s}: \Gamma(N) \rightarrow C$ and $K_{s}=\operatorname{ker}\left(L_{s}\right)$. Note that by construction $e_{i}^{s} \in K_{s}$ for all $i=1, \ldots 4$. We will prove Lemma 2.6 by finding suitable $g_{i}^{s}$ so that $L_{s}$ is surjective. Alternatively we can define a map

$$
F:[-1,1] \times V^{4} \rightarrow[-1,1] \times \operatorname{Hom}(K, C), \quad F\left(g_{1}^{s}, \ldots, g_{4}^{s}\right)=\left.L_{s}\right|_{K}
$$

and we need to show that the image of $F$ contains a family of surjective homomorphisms.

Lemma 2.7. Suppose that $g_{i}^{s}$ are given so that $\operatorname{dim}\left(K_{s}\right) \leq p+4$ for some $0 \leq p \leq m$ and all $s \in[-1,1]$. Then there exists a smooth family of linearly independent sections $\left\{f_{j}^{s}\right\}_{j=1}^{m} \in K$ that are orthogonal to $e_{i}^{s}$ for $i=1, \ldots, 4$ such that $\left\{f_{j}^{s}\right\}_{j=p+1}^{m} \in K_{s}^{\perp} \cap K$ for all $s$, and $<L_{s}\left(f_{j}\right), L_{s}\left(f_{k}\right)>=0$ for all $j \leq p$ and $k>p$.

Proof. Define $S_{p+4} \subset S_{p+3} \subset \ldots \subset S_{4}=[-1,1]$ where $S_{r}=\left\{s \in[-1,1] \mid \operatorname{dim}\left(K_{s}\right) \geq r\right\}$. Restricted to $S_{p+4}$ the vector spaces $K_{s}^{\perp} \cap K$ form a smooth vector bundle and so admit a smooth frame of $m-p$ sections $\left\{f_{j}^{s}\right\}_{j=p+1}^{m}$. But suppose that we have defined an $m-p$-frame (that is, $m-p$ linearly independent sections) over $S_{r}$. The vector spaces $K_{s}^{\perp} \cap K$ also form a continuous vector bundle over $S_{r-1} \backslash S_{r}$ which extend to a continuous vector bundle over $S_{r}$. Thus by the Tietze extension theorem the sections $\left\{f_{j}^{s}\right\}_{j=p+1}^{m}$ extend to $S_{r-1}$ and we can conclude by induction to define these sections over $[-1,1]$. The other sections $f_{j}^{s}$ can then be constructed smoothly over $s$ since the orthogonal complement of the sections already constructed forms a vector bundle over $[-1,1]$ which therefore admits a smooth frame field. We can arrange that their images are othogonal by a Gram-Schmidt procedure.

Lemma 2.8. Let $\hat{K} \subset K$ and $\hat{C} \subset C$ be p-dimensional vector spaces. Then the map

$$
\hat{F}: V^{4} \rightarrow \operatorname{Hom}(\hat{K}, \hat{C}), \quad \hat{F}\left(g_{1}, \ldots g_{4}\right)=\left.\pi_{\hat{C}} \circ Y\right|_{\hat{K}},
$$

where $Y: N \rightarrow \Lambda^{0,1}\left(T^{*} S \otimes_{\mathbb{C}} N\right)$ is the homomorphism associated to the $g_{i}$, is nonzero.

Proof. Let $a_{i j}$ be such that $f_{j}^{s}=\sum_{i=1}^{4} a_{i j}^{s} e_{i}^{s}$ on $U$. Then $\hat{F}\left(g_{1}, \ldots g_{4}\right)\left(f_{j}^{s}\right)=\sum_{i=1}^{4} g_{i}^{s} a_{i j}^{s}$ on $U$.

If $\hat{F}$ was trivial, then the $L^{2}$ inner product

$$
\sum_{i=1}^{4}\left\langle g_{i}^{s} \cdot a_{i j}^{s}, \eta_{k}\right\rangle=0 \quad \forall k, j \quad \forall g_{i}^{s} \in V
$$

Thus $\left(a_{i j}^{s} \eta_{k}\right)_{i=1}^{4} \in\left(V^{4}\right)^{\perp}$ for all $j, k$. Recall that $K$ and $C$ are the kernel and cokernel of a real-linear Cauchy-Riemann operator $D$, respectively. Then for fixed $j, k$, if $a_{i j}^{s} \eta_{k} \in V^{\perp}$ for all $i$, we have over $U$

$$
D^{*}\left(a_{i j}^{s} \eta_{k}\right)=\left(\bar{\partial}^{*} a_{i j}^{s}\right) \otimes \eta_{k}=0 \quad \forall i
$$


On the other hand

$$
0=D f_{j}=\sum_{i=1}^{4} D\left(a_{i j}^{s} e_{i}^{s}\right)=\sum_{i=1}^{4}\left(\bar{\partial} a_{i j}^{s}\right) \otimes e_{i}^{s}
$$

and the two equations taken together imply that the $\left\{a_{i j}^{s}\right\}_{i=1}^{4}$ are constant functions over $U$. This means that $f_{j}^{s}$ is a linear combination of the $e_{i}^{s}$ on $U$. By unique continuation, using that $f_{i}^{s}$ and $e_{i}$ are in the kernel of the operator $D$, we conclude that $f_{j}^{s}$ is globally a linear combination of the $e_{i}$. But that contradicts that $f_{j}^{s}$ is linearly independent of the $e_{i}^{s}$.

Lemma 2.9. Suppose that $Y_{s}$ and corresponding sections $f_{j}$ are given as in Lemma 2.7. Then there exist a smooth family $\left(g_{i}^{s}\right)_{i=1}^{4} \in V^{4}$ such that for each of the corresponding maps $L_{s}$ there exists an $f^{s} \in \operatorname{span}\left\{f_{j}^{s}\right\}_{j=1}^{p}$ with $L_{s}\left(f^{s}\right) \neq 0$ and independent of the span of the $L_{s}\left(f_{j}\right)$ for all $j>p$.

Proof. As in the previous lemma we express the $\left\{f_{j}^{s}\right\}_{j=1}^{p}$ as a linear combination of functional multiples of the $\left\{e_{i}^{s}\right\}_{i=1}^{4}$, at least over the open set $U$. Then we redefine the above linear map $F$ as in Equation (4) such that its range is paths of $p \times p$ matrices with entries determined by the $L^{2}$ inner products of the $\left\{f_{j}^{s}\right\}_{j=1}^{p}$ with $\left\{\eta_{j}^{s}\right\}_{j=1}^{p}$ orthogonal to the $L_{s}\left(f_{j}\right)$ for all $j>p$. This linear map is nonzero for all $s$ by Lemma 2.8. Therefore it has a positive codimensional kernel $M^{s} \subset V^{4}$ for all $s$ and the lemma follows if we can find a continuous section of $M^{\perp}$ over $[0,1]$. But the rank of these vector spaces is again lower semicontinuous in $s$ and so does indeed admit a section as above by first defining over points of minimal rank and then extending as before in the proof of Lemma 2.7.

Proof of Lemma 2.6. We prove this by induction by perturbing $g_{i}^{s}$. Suppose that we have found $g_{i}^{s}$ such that $\operatorname{dim} \operatorname{ker}\left(K_{s}\right) \leq p+4$ for all $s$ and some $0<p \leq m$. Then we can apply Lemma 2.7 to find corresponding families of sections and thus a perturbation of the $g_{i}^{s}$ using Lemma 2.9. If the $g_{i}^{s}$ are chosen sufficiently small then the $L_{s}\left(f_{j}\right)$ are still linearly independent for all $s$ and $j=p+1, . ., m$. However for each $s$ there is now an $f^{s} \in \operatorname{span}\left(\left\{f_{j}^{s}\right\}_{j=1}^{p}\right)$ with $L_{s}\left(f^{s}\right) \neq 0$ and independent to the $L_{s}\left(f_{j}\right)$ for $j>p$. Hence for each $s$ we have that $\operatorname{dim}\left(K_{s}\right) \leq p+4-1$ and the proof follows.

Let $Y_{s}$ and $L_{s}$ be as in Lemma 2.6 and consider the family of real-linear Cauchy-Riemann operators

$$
D_{s, t}=D+t Y_{s} \text {. }
$$

The kernel of $D_{s, t}$ gets arbitrarily close to $K_{s}$ as $t$ gets small as described below. This result is well established in the literature (see e.g. [Kat95]), but we give a proof here for the convenience of the reader. In the following $\|\cdot\|$ denotes the $L^{2}$-norm.

Lemma 2.10. There exists a constant $c>0$ so that for all $0<|t|<1 / 2 c, s \in[-1,1]$ and $v_{s} \in K_{s}, D_{s, t}$ is surjective and there exists a unique $\xi_{s, t}\left(v_{s}\right) \in K_{s}^{\perp}$ so that

$$
v_{s}+\xi_{s, t}\left(v_{s}\right) \in \operatorname{ker} D_{s, t} .
$$

Moreover $\left\|\xi_{s, t}\left(v_{s}\right)\right\| \leq 2 t C\left\|v_{s}\right\|$. 
Proof. Let $V_{s}=K_{s}^{\perp} \cap K$ denote the orthogonal complement of $K_{s}$ in $K$ and set

$$
\tilde{L}_{s}=\left.L_{s}\right|_{V_{s}}: V_{s} \rightarrow C .
$$

Then $\tilde{L}_{s}$ is an isomorphism.

Let $W_{s}=K_{s}^{\perp} \cap \operatorname{ker} L_{s} \subset L^{2}(N)$ and consider the compact operator

$$
F_{s}: \operatorname{ker} L_{s} \rightarrow W_{s}, \quad F_{s}(\zeta)=\tilde{L}_{s}^{-1} \circ L_{s}\left(P Y_{s}(\zeta)\right)-P Y_{s}(\zeta)
$$

where $P: C^{\perp} \rightarrow K^{\perp}$ is the inverse of $\left.D\right|_{K^{\perp}}: K^{\perp} \rightarrow C^{\perp}$.

Note that $L_{s} \circ F_{s}=0$ and $\tilde{L}^{-1}$ and $P$ have image in $K_{s}^{\perp}$, so $F_{s}$ is well defined. Let $c=\sup _{s \in[-1,1]}\left\|F_{s}\right\|_{L^{2}}$. For $|t| c \leq \frac{1}{2}$ and $v \in \operatorname{ker} L_{s}$ note that

$$
\left\|\sum_{n=1}^{N} t^{n} F_{s}^{n}(v)\right\| \leq \sum_{n=1}^{N}|t|^{n}\left\|F_{s}^{n}(v)\right\| \leq \sum_{n=1}^{N}|t|^{n} c^{n}\|v\|<2|t| c\|v\|
$$

so we may define

$$
\xi_{s, t}(v)=\sum_{n=1}^{\infty} t^{n} F_{s}^{n}(v)
$$

also satisfying $\left\|\xi_{s, t}(v)\right\| \leq 2 t c\|v\|$. Moreover,

$$
D_{s, t} \circ F_{s}=D \circ F_{s}+t Y_{s} \circ F_{s}=-Y_{s}+t Y_{s} \circ F_{s}
$$

and thus

$$
\begin{aligned}
D_{s, t}\left(v+\sum_{n=1}^{N} t^{n} F_{s}^{n}(v)\right) & =D v+t Y_{s}(v)+\sum_{n=1}^{N}\left(t^{n+1} Y_{s} F_{s}^{n}(v)-t^{n} Y_{s} F_{s}^{n-1}(v)\right) \\
& =D v+t^{N+1} Y_{s} F_{s}^{N}(v),
\end{aligned}
$$

which converges strongly to $D v\left(\right.$ in $L^{2}$ ) as $N \rightarrow \infty$, so

$$
D_{s, t}\left(v+\xi_{s, t}(v)\right)=D v, \quad \forall v \in \operatorname{ker} L_{s} .
$$

In particular $v+\xi_{s, t}(v) \in \operatorname{ker} D_{s, t}$ for all $v \in K_{s}$.

Next we show that $D_{s, t}$ is surjective. It suffices to show that the image of $D_{s, t}$ is dense as $D_{s, t}$ is Fredholm and thus has a closed image. By Hahn-Banach, it suffices to show that there does not exists $0 \neq \mu \in L^{2}\left(\Lambda^{0,1}\left(T^{*} S^{2} \otimes_{\mathbb{C}} N\right)\right)$ that annihilates the image of $D_{s, t}$. Suppose to the contrary such a $\mu$ exists. Write $\mu=\mu_{0}+\mu_{1}$, where $\mu_{0} \in C$ and $\mu_{1} \in C^{\perp}$. Without loss of generality assume that $\mu_{1} \neq 0$, otherwise, for $\zeta=\tilde{L}_{s}^{-1}\left(\mu_{0}\right) \in V_{s}$,

$$
\left\langle D_{s, t} \zeta, \mu\right\rangle=\left\langle t Y_{s}\left(\tilde{L}_{s}^{-1}\left(\mu_{0}\right)\right), \mu_{0}\right\rangle=t\left\langle\tilde{L}_{s}\left(\tilde{L}_{s}^{-1}\left(\mu_{0}\right)\right), \mu_{0}\right\rangle=t\left\|\mu_{0}\right\|^{2}=t\|\mu\|^{2} \neq 0 .
$$

Set $\zeta=P\left(\mu_{1}\right)-\tilde{L}_{s}^{-1} \circ L_{s} \circ P\left(\mu_{1}\right) \in \operatorname{ker} L_{s}$ and consider $\zeta+\xi_{s, t}(\zeta)$. Then

$$
\left\langle D_{s, t}\left(\zeta+\xi_{s, t}(\zeta)\right), \mu\right\rangle=\langle D \zeta, \mu\rangle=\left\langle\mu_{1}, \mu\right\rangle=\left\|\mu_{1}\right\|^{2} \neq 0 .
$$

This shows that $D_{s, t}$ is surjective.

The uniqueness of $\xi_{s, t}(v)$ satisfying $D_{s, t}\left(v+\xi_{s, t}(v)\right)$ follows from the surjectivity of $D_{s, t}$. 
In particular the above Lemma guarantees that for any given $\delta>0$ there exists $t_{0}>0$ so that for all $t<t_{0}$ the regular operators $D_{s, t}$ are superregular for $s \in[\delta, 1]$ and $D_{s, t}$ are not superregular for $s \in[-1,-\delta]$. To see this note that for $s$ in that range the quantity $e_{4}^{s}=s e_{4}+(1-s) e_{5}$ satisfies $\left\langle e_{4}(0), e_{4}^{s}(0)\right\rangle<0$ and $\left\langle e_{4}\left(p_{0}\right), e_{4}^{s}\left(p_{0}\right)\right\rangle=1$ by Equation (3). Thus near $p_{0}$ the tuple $\left(e_{1}, e_{2}, e_{3}, e_{4}^{s}\right)$ forms an oriented basis of $\mathbb{R}^{4}$ and at the point 0 they form a basis with the opposite orientation. In particular there must be points in $S^{2}$ where the sections do not form a basis of $\mathbb{R}^{4}$. This remains true under small perturbations of the tuple $\left(e_{1}, e_{2}, e_{3}, e_{4}^{s}\right)$.

A real-linear Cauchy Riemann operator $D$ on $N$ gives rise to an $\mathbb{R}$-invariant almost complex structure $J$ on the total space of $N$ in the following way. Choose a local complex trivialization $N=S^{2} \times \mathbb{C}^{2}$ and write $D=\bar{\partial}_{0}+\frac{1}{2} Y \circ j$, where $Y \in \operatorname{Hom}_{\mathbb{R}}\left(\mathbb{C}^{2}, \Lambda^{0,1} T^{*} S^{2} \otimes \mathbb{C}^{2}\right)$. Utilizing the projections to each factor $S^{2}$ and $\mathbb{C}^{2}$ of $N$, referred to as the horizontal and vertical directions with complex structures $j$ and $i$, respectively, we define the almost complex structure $J$ at a point $x=(w, u) \in N$ acting on a vector $(h, v) \in T_{x} N$ via

$$
J(h, v)=j h+Y_{(w, u)} h+i v .
$$

Note that $J$ is independent of the trivialization chosen and indeed satisfies $J^{2}=-$ Id. Moreover, if $f: S^{2} \rightarrow N$ with $f(z)=(w(z), u(z)) \in S^{2} \times \mathbb{C}^{2}$ in the homology class of a section, then

$$
\bar{\partial}_{J} f=\frac{1}{2}\left\{d f+J d f \circ j_{0}\right\}=\frac{1}{2}\left\{d w+j d w \circ j_{0}\right\}+\frac{1}{2}\left\{d u+i d u \circ j_{0}+Y_{(w, u)} d w \circ j_{0}\right\}
$$

Thus for $\bar{\partial}_{J} f=0$ it is necessary that $w(z)=z$ and $j=j_{0}$, up to a diffeomorphism of the domain $S^{2}$. In that case

$$
\bar{\partial}_{J} f=\frac{1}{2}\left\{d u+i d u \circ j_{0}+Y_{(w, u)} \circ j\right\}=D(u)
$$

so maps $f: S^{2} \rightarrow N$ in the class of a section are $J$-holomorphic if and only if they can be parametrized as a section $f(z)=(z, \xi(z))$ and $D \xi=0$. Moreover note the the zero section is always a $J$-holomorphic section no matter what $D$ is and that the linearization of $\bar{\partial}_{J}$ at the zero section is $D$.

Let $\omega$ be the canonical product symplectic form on $N$ so that on each fiber it reduces to the Fubini-Study form and let $\tilde{J}$ be the canonical product complex structure on $N$ and $\tilde{D}$ the associated Cauchy-Riemann operator. Given any symplectic 4-manifold $\left(M, \omega_{M}\right)$ there exists a symplectic embedding from $U$ into $(X, \omega)=\left(S^{2} \times M, \sigma_{0} \oplus \omega_{M}\right)$ preserving the $S^{2}$ factors, where $U$ is a suitable small neighborhood of the zero-section in $N$. Thus $\tilde{J}$ extends to a product complex structure on $X$ which is tamed by $\omega$, and $X$ is smoothly foliated by regular $\tilde{J}$-holomorphic spheres.

Let $D_{s}, s \in[-1,2]$ be a smooth family of real-linear Cauchy Riemann operators on $N$ so that $D_{s}=D_{s, t}$ for some small fixed $t$ and $s \in[-1,1]$, where $D_{s, t}$ is the operator from Lemma 2.10, and $D_{s}$ interpolates between $D_{1}$ and $\tilde{D}$ for $s \in[1,2]$. Denote the associated family of almost complex structures by $J_{s}$. Note that $J_{s}$ are tamed by $\omega$ on a neighborhood $U$ of the zero section in $N$. We now modify the family $J_{s}$ to construct a family of almost complex structures $\tilde{J}_{s}$ on $N$ with the property that $\tilde{J}_{s}=\tilde{J}$ outside of $U$ and $\tilde{J}_{s}=J_{s}$ in an open neighborhood $V \subset U$ of the zero section so that $\tilde{J}_{s}$ is tamed by $\omega$. Using the above embedding we similarly construct the family $\tilde{J}_{s}$ on $X$. 
The family $\tilde{J}_{s}$ is tamed by the canonical symplectic structure on $X=S^{2} \times M$, and $\tilde{J}_{2}$ is the product complex structure on $X$. Thus $\tilde{J}_{2}$ is regular (and superregular) and $X$ is foliated by $\tilde{J}_{2}$-holomorphic spheres. By construction $\tilde{J}_{s}$ is regular for all curves outside of $U$ and inside of $V$. By possibly adding a small perturbation to the family $\tilde{J}_{s}$ over $U \backslash V \subset X$ we may assume that the family $\tilde{J}_{s}$ is a regular family of almost complex structure and that $\tilde{J}_{-1}$ is regular.

Since $\tilde{J}_{s}=\tilde{J}$ outside of $U$, the complement of $U$ is foliated by $\tilde{J}$-holomorphic spheres for all $s \in[-1,2]$. Moreover, the zero section is $\tilde{J}_{s}$-holomorphic for all $s \in[-1,2]$. But the linearized operator at the zero section is $D_{s}$, which is not superregular for $s=-1$ by construction, so the foliation does not persist to a $J_{-1}$-holomorphic foliation of $X$.

This proves Theorem 1.3 in the case that $(X, \omega)=\left(S^{2} \times M^{4}, \sigma_{0} \times \omega_{M}\right)$.

\section{Stability of Foliations for Integrable Complex Struc- tures}

In this section we show that holomorphic foliations are stable under perturbations of complex structure so that the holomorphic bisectional curvature (see below or e.g. [KN96]) remains bounded.

Definition 3.1. Let $(M, J)$ be a complex manifold. The holomorphic bisectional curvature $H\left(p, p^{\prime}\right)$ of two $J$-invariant planes $p$ and $p^{\prime}$ in $T_{x} M$ is

$$
H\left(p, p^{\prime}\right)=R(X, J X, Y, J Y)
$$

where $R$ is the regular Riemannian curvature tensor and $X$ and $Y$ are unit vectors in $p$ and $p^{\prime}$, respectively.

We say that $(M, J)$ has holomorphic bisectional curvature bounded from above (below) by a constant $c$ if $H\left(p, p^{\prime}\right) \leq c\left(H\left(p, p^{\prime}\right) \geq c\right)$ for all $x \in M$ and $J$-invariant planes $p, p^{\prime} \subset T_{x} M$.

The following result is a computation from page 79 of [GH78].

Lemma 3.2. Let $G \rightarrow M$ be a holomorphic vector bundle of (complex) rank at least 2 over a complex manifold $M$, and let $E \subset G$ be a holomorphic subbundle and $F=E^{\perp}$ the orthogonal complement of $E$ in $G$. Then the local curvature form of $F$ is greater than or equal to the curvature form of $G$ restricted to $F$.

Lemma 3.3. Let $(X, \omega, J)$ be Kähler and let $u: S^{2} \rightarrow X$ be a J-holomorphic sphere. Assume that the the holomorphic bisectional curvature of $(X, J)$ is bounded from below by $c>\pi k / \omega[u]$. Then the pullback bundle $u^{*} T X$ has no holomorphic line-subbundle with first Chern class less than or equal to $k$.

Proof. Let $F$ be a holomorphic line-subbundle of $u^{*} T X$, and let $E$ be a complementary holomorphic subbundle, which exists by a result of Grotherndieck [Gro57] if the real dimension of $X$ is at least 4 and is taken to be empty otherwise. Let $E^{\perp}$ denote the orthogonal complement of $E$ in $u^{*} T X$ and denote the curvature of $E^{\perp}$ with respect to the connection induced by $u^{*} T X$ by $K$. By Lemma 3.2 we know that $K(\cdot) \geq u^{*} H(\cdot, F)$ on any complex frame. Then

$$
c_{1}(F)=c_{1}\left(E^{\perp}\right)=\frac{1}{2 \pi} \int_{S^{2}} K \geq \frac{1}{2 \pi} \int_{S^{2}} u^{*} H(\cdot, F) \geq \frac{1}{2 \pi} c \int_{S^{2}}\|d u\|^{2} d \mathrm{vol}>k .
$$


Recall Definition 1.5 for our use of the terms regular and superregular.

Lemma 3.4. Let $(X, \omega, J)$ be Kähler so that the holomorphic bisectional curvature is bounded from below by $c>-2 \pi / \omega(A)$, where $A \in H_{2}(X ; \mathbb{Z})$. Then any $J$-holomorphic sphere in the class of $A$ is regular.

If furthermore $u$ is immersed, $c_{1}(A)=2$, and the holomorphic bisectional curvature is bounded from below by $c>-\pi / \omega(A)$, then $u$ is also superregular.

Proof. Let $u: S^{2} \rightarrow X$ be a $J$-holomorphic curve representing $A$. By Lemma 3.3, and using that $c>-2 \pi / \omega(A)$, the pullback tangent bundle $u^{*} T X$ does not have a holomorphic linesubbundle with first Chern class less than -1 . Thus $u$ is regular by Lemma 3.3.1 in [MS04.

If furthermore $u$ is immersed and $c>-\pi / \omega(A)$, then every holomorphic line-subbundle of the normal bundle has first Chern class $\geq 0$. Since $u$ is immersed and $c_{1}(A)=2$, the first Chern class of the normal bundle is 0 . Thus any holomorphic line-subbundle of the holomorphic normal bundle has first Chern class 0 and has a superregular basis. So the normal bundle to $u$ has a superregular basis and $u$ is superregular.

Corollary 3.5. Let $J_{I}^{c}$ be the space of integrable compatible complex structures on $\left(X^{2 n}, \omega\right)$ with holomorphic bisectional curvature bounded from below by $c>-2 \pi / \omega(A)$, where $A \in$ $H_{2}(X ; \mathbb{Z})$ with $c_{1}(A)=2$. Further assume that there exists a $J_{0}$-holomorphic foliation of $X$ by spheres in the class of $A$ for some $J_{0} \in \mathcal{J}_{I}^{c}$.

Then $X$ is foliated by $J$-holomorphic spheres for any $J \in \mathcal{J}_{I}^{c}$ in the path-component of $J_{0}$.

Proof. Let $J=J_{1} \in \mathcal{J}_{I}^{c}$ be connected to $J_{0}$ via a path $\left\{J_{t}\right\}_{t \in[0,1]}$ and let $\mathcal{M}$ denote the family space of $\left\{J_{t}\right\}_{t \in[0,1]}$-holomorphic spheres in the class of $A$ as in Equation (11) and let

$$
\mathcal{M}^{1}=\mathcal{M} \times_{G} S^{2}
$$

denote the component of the one-pointed moduli space, modulo automorphisms.

By Lemma 3.4 all curves in $\mathcal{M}^{1}$ are regular, so $\mathcal{M}^{1}$ is a smooth manifold (of dimension $2 n+1)$ and the projection onto the $[0,1]$-factor is a submersion.

Denote the connected component of $\mathcal{M}^{1}$ containing the initial $J_{0}$-holomorphic foliation by $\tilde{M}$ and let $\tilde{M}_{s}=\{(u, p, t) \in \tilde{M} \mid t=s\} \subset \mathcal{M}$. Again, by Lemma 3.4, all curves in $\mathcal{M}_{s}$ are regular, so $\mathcal{M}_{s}$ is a smooth manifold (of dimension $2 n$ ). The evaluation map $e v_{s}: \mathcal{M}_{s} \rightarrow X$ is holomorphic with respect to the natural complex structure on $\mathcal{M}_{s}$. It has degree 1 , since $e v_{0}$ is of degree 1 by assumption. Thus $e v_{s}$ is a diffeomorphism for all $s \in[0,1]$ and $\mathcal{M}_{s}$ is a smooth foliation of $X$.

Remark 3.6. Note that any $J$-holomorphic sphere $u$ (for integrable $J$ ) that is part of a smooth foliation is automatically regular and superregular. Indeed, any line subbundle of the normal bundle of $u$ has non-negative first Chern class, since it has holomorphic sections induced by nearby curves. Since the first Chern class of the normal bundle at a leaf of a foliation is trivial all linear subbundles must have first Chern class 0 , so the curve is regular and superregular.

Under more stringent curvature assumptions, we can prove the existence of foliations given conditions on a Gromov-Witten invariant. This is the substance of Theorem 1.4 that we are now prepared to prove. 
Proof of Theorem 1.4. Let $J \in \mathcal{J}_{I}^{c}$ and let $\mathcal{M}$ be the space of $J$-holomorphic spheres in the class $A$. $\mathcal{M}$ is non-empty since the GW count is non-zero. Let $u \in \mathcal{M}$. By Lemma 3.3 we know that all holomorphic linear subbundles of $u^{*} T X$ have first Chern class greater than or equal to 0 and $u$ is regular. We claim that $u$ is immersed. If not, then the first Chern class of the tangent bundle is at least 4 . But by the dimension formula we know that $c_{1}(A)=2$, so the holomorphic normal bundle would contain a linear subbundle with first Chern class less than 0 which is impossible. Thus $u$ is superregular.

Since every $u \in \mathcal{M}$ is regular and superregular, the evaluation map is transverse to any $x \in X$ and the curves contribute positively to the GW count of a point class. Thus the evaluation map $e v: \mathcal{M} \rightarrow X$ has degree one and is holomorphic, so it is a diffeomorphism, showing that $X$ is foliated by embedded holomorphic spheres.

\section{References}

[AM00] Miguel Abreu and Dusa McDuff, Topology of symplectomorphism groups of rational ruled surfaces, J. Amer. Math. Soc. 13 (2000), no. 4, 971-1009 (electronic). MR MR1775741 (2001k:57035)

[Don02] S. K. Donaldson, Holomorphic discs and the complex Monge-Ampère equation, J. Symplectic Geom. 1 (2002), no. 2, 171-196. MR MR1959581 (2003m:32037)

[GH78] P Griffiths and J. Harris, Principles of algrebraic geometry, John Wiley \& Sons, 1978.

[Gro57] A. Grothendieck, Sur la classification des fibrés holomorphes sur la sphère de Riemann, Amer. J. Math. 79 (1957), 121-138. MR MR0087176 (19,315b)

[Gro85] M. Gromov, Pseudo holomorphic curves in symplectic manifolds pseudo holomorphic curves in symplectic manifolds, Invent. Math. 82 (1985), no. 2, 307-347.

[Hin04] R. Hind, Lagrangian spheres in $S^{2} \times S^{2}$, Geom. Funct. Anal. 14 (2004), no. 2, 303318. MR MR2060197 (2005g:53151)

[Hir59] Morris W. Hirsch, Immersions of manifolds, Trans. Amer. Math. Soc. 93 (1959), 242-276. MR MR0119214 (22 \#9980)

[Kat95] Tosio Kato, Perturbation theory for linear operators, Classics in Mathematics, Springer-Verlag, Berlin, 1995, Reprint of the 1980 edition. MR MR1335452 (96a:47025)

[KN96] Shoshichi Kobayashi and Katsumi Nomizu, Foundations of differential geometry. Vol. II, Wiley Classics Library, John Wiley \& Sons Inc., New York, 1996, Reprint of the 1969 original, A Wiley-Interscience Publication. MR MR1393941 (97c:53001b)

[MS04] D. McDuff and D. Salamon, J-holomorphic curves and symplectic topology, 2 ed., American Mathematical Society, 2004.

[Tau96] Clifford H. Taubes, SW $\Rightarrow$ Gr: from the Seiberg-Witten equations to pseudoholomorphic curves, J. Amer. Math. Soc. 9 (1996), no. 3, 845-918. MR MR1362874 (97a:57033) 